\title{
INFERIORLY BASED NASOLABIAL FLAP: A RELIABLE OPTION FOR ORAL AND MAXILLOFACIAL RECONSTRUCTION
}

\author{
Mohamed Koraitim*, Aly M. Atteya * and Shady A. Hassan**
}

\begin{abstract}
Introduction: Reconstruction of defects in the maxillofacial region can be challenging. Anatomy, location and size of the defect are major determinants of type of flap that could be used whether local, regional or distant. Nasolabial flap is one of the local flaps that can be used for reconstructing small to moderate size soft tissue defects, especially when microsurgical free flaps are less feasible due to lack of infrastructure, expertise or financial constraints.
\end{abstract}

Aims and Objectives: To study the effectiveness of the nasolabial flaps in reconstruction of orofacial defects of various origins.

Materials and Methods: A prospective study was conducted on 37 patients (49 nasolabial flaps) 25 unilateral flaps \& 12 bilateral flaps. Of these, 30 patients had reconstruction following oncological resection. All flaps were inferiorly based, and 36 flaps required a second stage for separation after 3 weeks.

Patients were evaluated for postoperative complications, flap uptake, donor site morbidity, postoperative extraoral scarring and patient's satisfaction.

Results: Good results in form of function and cosmesis were obtained in most of patients. Postoperative complications were relatively minor as tip of flap necrosis, and oro-cutaneous fistula. The average time taken for flap harvesting was 43 minutes for bilateral nasolabial flaps and 27 minutes for unilateral ones.

Conclusion: The nasolabial flap is a simple and reliable flap that can be used in reconstruction of small to moderate sized defects in orofacial region with good cosmetics and functional outcomes.

KEYWORDS: Nasolabial, flap, reconstruction.

* Consultant and Lecturer of Maxillofacial and Plastic Surgery, Faculty of Dentistry, Alexandria University.

** Maxillofacial and Plastic Surgery, Faculty of Dentistry, Alexandria University. 


\section{INTRODUCTION}

Reconstruction of maxillofacial defects is challenging because of the complexity of this area where a small or medium sized defect can result in cosmetic and functional comorbidities to the patient. ${ }^{1}$ Maxillofacial defects can result following tumor resection, traumatic injuries, or infection. Reconstructive options depend on the size, site, tissue components, and patient's general condition. They range from simple closure, skin grafts, local or regional flaps to more complex distant microvascular flaps. The latter is considered the only available option in large, composite defects to achieve the best cosmetic and functional outcomes. ${ }^{2}$

Many local and regional flaps are available and considered a safe and useful alternative to distant flaps such as the mucosal cheek flap, Buccal fat pad flap, tongue flap, buccinators myomucosal flap, facial artery myomucosal flap, temporalis muscle flap and nasolabial flap. ${ }^{3}$

Nasolabial flap has a wide scale for usage as it can be used for the reconstruction of the nose, lips and intraoral defects either separately or together due to their close boundaries. ${ }^{4}$

In our research we have used inferiorly based nasolabial flap for reconstructing a wide variety of small to moderate size defects and we evaluated the cosmetic and functional outcomes such as eating and speech.

\section{MATERIAL AND METHODS}

Between January of 2016 and of May 2018， 49 inferiorly based nasolabial flaps were used as a method for reconstructing small to moderate size soft tissue defects in the orofacial region in the maxillofacial departments, Alexandria University, Egypt.

This prospective observational clinical study involved 37 patients that were selected randomly regardless of age, and sex.

\section{Inclusion Criteria (Fig.1)}

1. Patients with small to moderate size orofacial soft tissue defect not exceeding $2.5 * 6.5 \mathrm{~cm}$.

2. Elderly Patients with larger defects than $2.5 * 6.5$ $\mathrm{cm}$ with comorbidities conflicts with lengthy operations.

\section{Exclusion Criteria}

1. Small defects that can be closed primarily with direct closure methods.

2. Large defects that its reconstruction needs another flap with larger surface area more than $2.5 * 6 \mathrm{~cm}$.

3. Bony defects which are better reconstructed by osseous flaps.

Demographic data, cause, site, and size of the defect, size of the flap, and time to harvest it were collected. Success of reconstruction and complications were recorded.

\section{Surgical Technique (Fig 2)}

After the surgical excision in case of oncologic resection, we began with marking of the nasolabial flap using Methylene blue solution before injecting local anesthetic with epinephrine into the flap to avoid distortion of the flap anatomy.

Appropriate measures are taken with a gauge to ensure that the flap is of adequate length and will rotate without kinking and minimum standing cutaneous deformity. The lateral dimensions of the flap are outlined for maximum cosmetic results. The width of the flap at the base is usually 1.5-2.5 $\mathrm{cm}$ depending on the nasolabial fold and the cheek adjacent to it, and maximum tissue is available just above the angle of the mouth. The length was kept at 5-7 cm. The medial and lateral limbs of the incision tapered together superiorly reaching below the medial canthus. Tapering of the flap avoided puckering or standing cutaneous deformity 


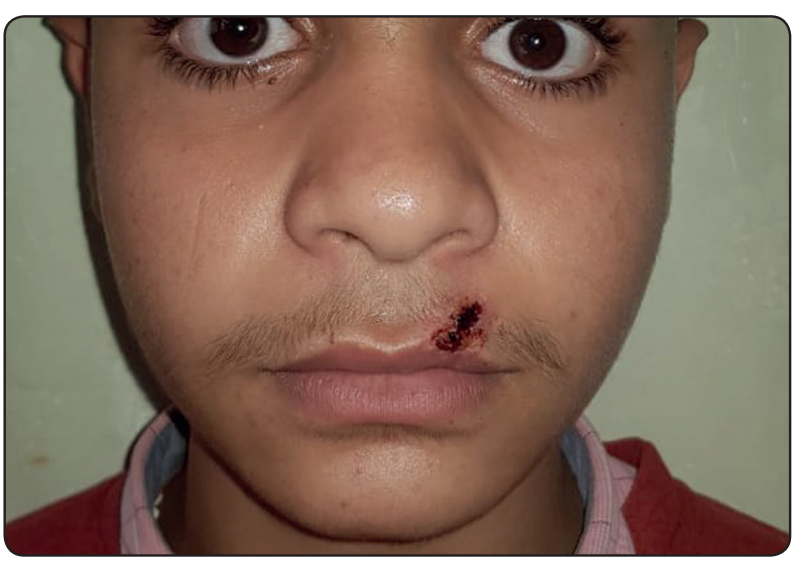

Fig. (1) preoperative image of patient with upper lip basal cell carcinoma.

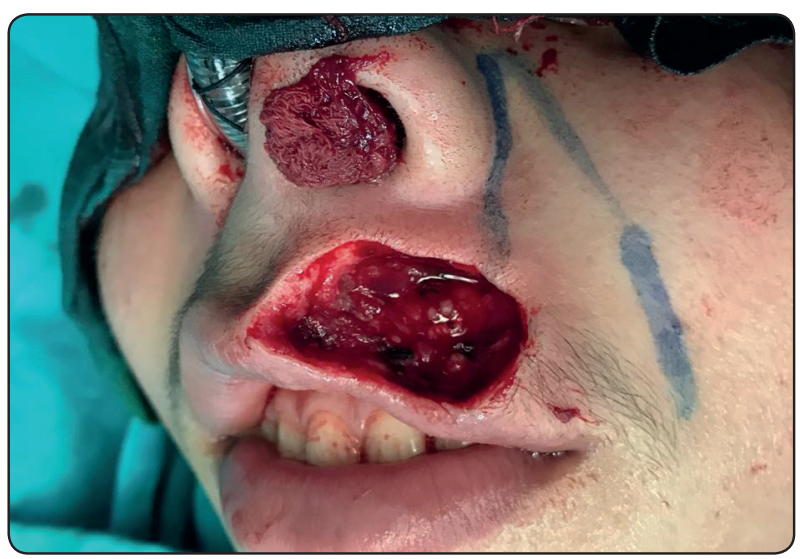

Fig. (2) upper lip defect and marking of the nasolabial flap.

formation in the closure of the donor nasolabial area. We started with meticulous manipulation and dissection of the flap from superior to inferior in a supramuscular plane using dissecting scissors.

When used intraorally, a transbuccal tunnel is created with dissecting scissors at the level of the lateral commissure of the mouth $2 \mathrm{~cm}$ away from the corner of the mouth. The caudal base of the flap was de-epithelized in a rectangular fashion for a one-stage operation as there is no need for a pedicle detachment later. In two-staged flap reconstruction the secondary defect is closed by direct suturing, and a small triangular area is left unsutured near the base of the flap to avoid constriction of the base. Three weeks postoperatively the pedicles are divided, and the base is returned and sutured in its original place.

The flap was then transferred to oral cavity in a tension free manner and inserted into the defect with a series of simple interrupted sutures using Vicryl sutures (3-0 or 4-0)

Direct closure for the donor site was done into layers Vicryl 3-0 sutures for the deeper layer and Prolene 6-0 for final skin closure.

\section{Follow-up care}

Good monitor for the flap edges for viability was done in the first 72 hours postoperatively.

Good oral hygiene measures were instructed to the patients for intraoral reconstruction defects.

Objective measures for functional and cosmetics outcomes were done in form of assessment of speech, eating and aesthetics.

\section{RESULTS (Fig. 3)}

This study included 37 patients, 28 were males and 9 were females. The patients' age ranged from 16 to 71 years, with the majority being in the age group of 40- 60 years (mean age $=50.9$ years).

We used the flap to reconstruct defects following oncologic resection, post-traumatic defects and osteomyelitis debridement in 30,6 , and one patient, respectively.

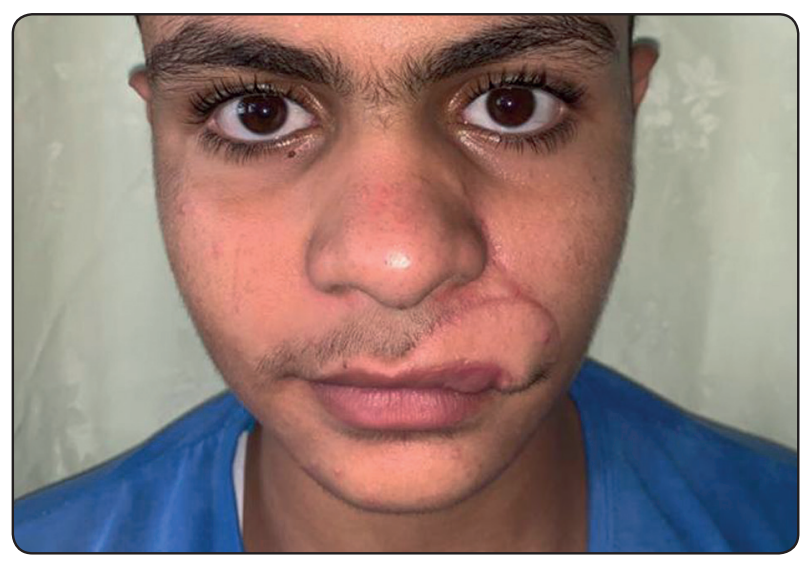

Fig. (3) long term follow-up of upper lip reconstruction with nasolabial flap. 
49 flaps were harvested, where the flap was harvested unilaterally in 25 patients and bilaterally in 12 patients. The location of the defects was in the floor of the mouth ( $\mathrm{n}=8$ patients), tongue defect ( $\mathrm{n}=12$ patients), combined defects for tongue and floor of the mouth ( $n=2$ patients), the lower lip $(n=3$ patients), the upper lip $(n=5)$, buccal mucosa $(\mathrm{n}=3)$, ala of the nose $(\mathrm{n}=2)$, and the collumella $(\mathrm{n}=2)$. The largest defect reconstructed was $3 \times 8 \mathrm{~cm}$ after partial glossectomy. The smallest defect was $1 \times 0.5 \mathrm{~cm}$ in the collumella.

All demographic data concerning the site of the defect, size, and time of harvest of the flap were collected in Table 1 .

Table (1) Demographic data of study patient

\begin{tabular}{|c|c|c|c|c|c|c|c|}
\hline $\begin{array}{l}\text { Patient } \\
\text { no }\end{array}$ & Sex & $\begin{array}{l}\text { Age by } \\
\text { years }\end{array}$ & $\begin{array}{l}\text { Dimensions } \\
\text { of } \\
\text { Defect, }{ }^{*} \mathrm{~cm}\end{array}$ & Location & $\begin{array}{l}\text { Unilateral or } \\
\text { bilateral flaps }\end{array}$ & $\begin{array}{c}\text { Time of } \\
\text { harvest/ } \\
\text { Min. }\end{array}$ & $\begin{array}{c}\text { Follow-up } \\
\text { Period, } \\
\text { By months }\end{array}$ \\
\hline 1 & M & 55 & $3 * 7$ & Floor of the mouth & Bilateral & 45 & 7 \\
\hline 2 & M & 54 & $2 * 5.5$ & Tongue & Unilateral & 25 & 9 \\
\hline 3 & $\mathrm{~F}$ & 43 & $3 * 1$ & Lower lip & Unilateral & 27 & 14 \\
\hline 4 & M & 16 & $2.5 * 2$ & Upper lip & Unilateral & & 13 \\
\hline 5 & $\mathrm{~F}$ & 38 & $2.5 * 6$ & Tongue and Floor of the mouth & Bilateral & 50 & 7 \\
\hline 6 & M & 62 & $3 * 5$ & Cheek and upper lip & Unilateral & 33 & 8 \\
\hline 7 & M & 30 & $1 * 0.5$ & Collumella & Unilateral & 28 & 8 \\
\hline 8 & $\mathrm{~F}$ & 53 & $2 * 6$ & Floor of the mouth & Bilateral & 45 & 6 \\
\hline 9 & M & 48 & $1.5 * 4$ & Tongue & Unilateral & 27 & 7 \\
\hline 10 & M & 52 & $2 * 6.5$ & Floor of the mouth & Bilateral & 39 & 9 \\
\hline 11 & M & 66 & $3 * 8$ & Tongue & Unilateral & 34 & 8 \\
\hline 12 & $\mathrm{~F}$ & 71 & $3 * 5$ & Floor of the mouth & Bilateral & 40 & 10 \\
\hline 13 & $\mathrm{~F}$ & 47 & $2.5 * 6.5$ & Tongue and Floor of the mouth & Bilateral & 55 & 6 \\
\hline 14 & M & 48 & $1.5 * 3$ & Upper lip & Unilateral & 18 & 18 \\
\hline 15 & M & 56 & $2 * 4$ & Tongue & Unilateral & 23 & 7 \\
\hline 16 & M & 59 & $2 * 6$ & Floor of the mouth & Bilateral & 38 & 13 \\
\hline 17 & $\mathrm{~F}$ & 43 & $1 * 2$ & Ala of the nose & Unilateral & 28 & 8 \\
\hline 18 & $\mathrm{~F}$ & 44 & $2 * 5$ & Tongue & Unilateral & 28 & 12 \\
\hline 19 & M & 56 & $4 * 5.5$ & Lower lip & Bilateral & 29 & 11 \\
\hline 20 & M & 61 & $3 * 4$ & Tongue & Unilateral & 33 & 10 \\
\hline 21 & $\mathrm{~F}$ & 38 & $1 * 1.5$ & Ala of the nose & Unilateral & 19 & 7 \\
\hline 22 & M & 54 & $5 * 3.5$ & Lower lip & Bilateral & 44 & 6 \\
\hline 23 & M & 47 & $1.5 * 3.5$ & Upper lip & Unilateral & 26 & 9 \\
\hline 24 & M & 53 & $2 * 4.5$ & Cheek & Unilateral & 29 & 10 \\
\hline 25 & M & 49 & $2 * 5$ & Tongue & Unilateral & 33 & 13 \\
\hline 26 & M & 52 & $1.5 * 6$ & Tongue & Unilateral & 38 & 6 \\
\hline
\end{tabular}




\begin{tabular}{|c|c|c|c|c|c|c|c|}
\hline 27 & $\mathrm{M}$ & 58 & $3 * 6$ & Floor of the mouth & Bilateral & 42 & 18 \\
\hline 28 & $\mathrm{M}$ & 48 & $1.5 * 3.5$ & Upper lip & Unilateral & 18 & 15 \\
\hline 29 & $\mathrm{M}$ & 49 & $3 * 5$ & Tongue & Unilateral & 25 & 18 \\
\hline 30 & $\mathrm{M}$ & 54 & $2.5 * 4$ & Floor of the mouth & Unilateral & 29 & 8 \\
\hline 31 & $\mathrm{M}$ & 52 & $2 * 5.5$ & Tongue & Unilateral & 24 & 14 \\
\hline 32 & $\mathrm{M}$ & 61 & $1.5 * 3$ & Upper lip & Unilateral & 19 & 11 \\
\hline 33 & $\mathrm{M}$ & 28 & $1.5 * .5$ & Collumella & Unilateral & 28 & 7 \\
\hline 34 & $\mathrm{~F}$ & 70 & $2 * 4.5$ & Tongue & Bilateral & 49 & 14 \\
\hline 35 & $\mathrm{M}$ & 62 & $3 * 6$ & Floor of the mouth & Unilateral & 28 & 16 \\
\hline 36 & $\mathrm{M}$ & 68 & $2.5 * 5.5$ & Tongue & Unilateral & 29 & 18 \\
\hline 37 & $\mathrm{M}$ & 49 & $2 * 3.5$ & Cheek & \\
\hline
\end{tabular}

All flaps were viable and functional outcome like speech and swallowing following tongue reconstruction and lip competence following lip reconstruction were good.

The average time taken for flap harvesting was 43 minutes for bilateral nasolabial flaps and 27 minutes for unilateral ones

Follow-up period ranged from 6-18 months with mean 9.6 months, Tip necrosis occurred in 8 flaps by 10-14 days post-operatively and it was managed conservatively; healed by secondary intention. Of these, 4 patients developed small orocutaneous fistula and were managed successfully by conservative measures.

12 patients had small area of hair growth at the reconstructed site (base of the flap) which disappeared in 8 patients after receiving postoperative radiotherapy.

None of our patients had ectropion and no other complications were noted during the entire followup period except for local recurrence in one patient after 9 months that was treated by further resection.

\section{Speech}

Twenty-four patients found their speech unchanged, seven patients said their speech had changed moderately and six patients notice much speech change after operation and postoperative radiotherapy especially in telephone communication.

No psychological impact was found except for the six patients that noticed change in telephone dialogue.

\section{Aesthetics}

Aesthetic outcome was judged as good by 18 patients and moderate by 11 . Two patients were not that satisfied. Six patients said there was little or no change in their appearance since the operation.

No patients mentioned that the new appearance obstacles their life activities.

\section{Eating}

Twenty three patients had little or no problems with the consumption of food.

Nine patients had considerable problems with masticating solid foods. Five

Patients did not have to adapt their meals.

Patients with prosthesis supported by dental implants experienced no mastication or eating problems after surgery. 


\section{DISCUSSION}

Oral and maxillofacial defects may be caused by benign and malignant lesions resection, traumatic soft tissue injuries and infections like osteomyelitis. Oral cancer resection can result in complex defects that are challenging to reconstruct. Even small to moderate size defects is crucial to reconstruct to avoid functional and cosmetic comorbidities to the patients and hence a better quality of life..$^{5,6}$

Reconstructive options for small to moderate defects of the orofacial region range from skin grafts, local flaps, regional flap up to free tissue microvascular flaps with its optimum reconstructive impact especially in composite defects. ${ }^{?}$

Skin grafting is a simple procedure and can be an option for reconstructing these defects; however it has certain drawbacks like contraction, abnormal pigmentations and high risk of graft failure especially when used in intraoral reconstruction because of possibility of saliva collection underneath. ${ }^{8,9}$

The pedicled temporalis muscle flap has its role for craniofacial and intraoral reconstruction especially retromolar area and cheek resection defects., The commonest problem with this flap is postoperative trismus which can be prevented by performing coronoidectomy that increase the operative time and adds morbidity at the operative site. ${ }^{10}$

We do believe that trismus is caused by fibrosis, especially after radiotherapy, in comparison with nasolabial flaps no trismus were encountered in any of our patients including those who received postoperative radiotherapy and this stands on for the rich blood supply of the nasolabial flap. ${ }^{11}$

A cosmetic drawback credited to the usage of temporalis muscle flap is the hollowness of the temple area which may need a porous polyethylene sheet to be used to fill the temporal fossa donor site. Therefore, the nasolabial flap is superior in being more economic reconstructive option and less liable to donor site complications as foreign body infections. ${ }^{11}$
Pectoralis major myocutaneous flap is rather considered a workhorse of maxillofacial reconstruction especially larger defects after floor of mouth excision or subtotal glossectomies. However, this bulky flap may be difficult to be inserted inside small to moderated defects and adds an extra soft tissue bulk in the neck. ${ }^{12}$

Regarding both temporalis and pectoralis major flaps both are quite distant flaps in comparison to nasolabial flaps which is a local flap requiring a shorter time to harvest. In our series the average time taken for flap harvesting was 43 minutes for bilateral nasolabial flaps and 27 minutes for unilateral ones making nasolabial flap a perfect reconstruction option for small to moderate size defects especially in patients with associated co-morbidities.

Free microvascular flaps can be the only reconstruction option in large and complex defects. Being a lengthy procedure requiring surgical expertise, economic resources and increase the hospital stay period, free flaps may not be a suitable option for many patients especially those with significant comorbidities. ${ }^{13}$

The skin of the nasolabial fold is nourished by multiple small branches from the superior labial artery, and the angular artery nears the dorsum of the nose. Further superiorly the skin is nourished by the infraorbital artery. Hence, it is possible to design both an inferiorly based nasolabial flap, with the facial artery as its pedicle, and a superiorly based nasolabial flap with the infraorbital and transverse facial arteries as its pedicle giving the nasolabial flap a great chance for healing with minimal contraction comparing to skin grafts as well a more competent reconstruction as well. ${ }^{14}$ In this study, all our flaps were harvested as random flap with good viability which can be explained by its rich subdermal vascular plexus.

We stand in solidarity with the results of Hofstra et.al that the nasolabial flap has superior results in floor of the mouth reconstruction with no limitation of tongue movements and good speech and eating 
outcomes with no postoperative bulkiness as it is a thin pliable flap which offer a versatile option for floor of the mouth reconstruction. ${ }^{15}$

In our series there was no conflict with receiving postoperative radiotherapy for oncologic resection patients. With no subsequent complications and our results co incident with Soh and Soo, in their series of 11 cases, observed that nasolabial flaps can be performed even in patients who have received radiotherapy and those who have undergone a radical neck dissection. However, they observed that radiotherapy and ligation of the facial artery have some effects on flap healing pattern, and this was not observed in our series. ${ }^{16}$

The maximum dimensions of the flap have been said to be $7 \times 1.5 \mathrm{~cm}$. our inclusion criteria to small and moderate defects with dimensions of $2.5 * 6 \mathrm{~cm} .{ }^{17}$, ${ }^{18}$ However, we exceeded this dimensions in some elderly patients with comorbidities conflicted with a more lengthy reconstructive option and their skin laxity was very helpful in flap harvesting and closure giving the nasolabial flap a valuable advantage in this patient's category.

Ioannides and Fossion presented their work with a preference to the two-stage procedure as they found that it is safer to ensure a better circulation for the flap. Also, they found that the de-epithelialized part of the pedicle which lies on the alveolar process will usually prevent the patient from wearing a denture and may lead to formation of inclusion cysts, due to the hair follicles. In the current study, the site of the defect was our decision guide whether a single or two-stage procedure to be done. When used for intraoral reconstruction, Flaps were harvested in single stage in buccal mucosa reconstruction through de-epithelialization at their base, which proved to be safe as all flaps were viable and showed an uneventful healing. We prefer doing a two-stage procedure in floor of the mouth and alveolar reconstruction as the hanging part of the flap will interfere with denture wearing.

Nasolabial flap is thin and pliable making it a perfect option for floor of mouth reconstruction as it will not interfere with tongue movements which aids in both function as speech and permit a good lingual vestibule cleaning of food debris by selftongue movements. Here, the flap was harvested bilaterally for a tension free reconstruction as it can reach just beyond the midline. Also, the risk of tip necrosis should be taken into consideration.

Minor complications of the nasolabial flap were cosmetic outcomes at the nasolabial fold, hair growth, tip flap necrosis and flap bulkiness. In our series tip flap necrosis occurred in 8 flaps by a percentage of $21 \%$ by $10-14$ days post-operatively and it was managed conservatively, our results goes with Herbert and Harrison consequence that the distal third of the nasolabial flap should be considered of random distribution, therefore, partial necrosis of the flap occurs when the flap is too long relative to the base. In another results, Mutimer and Poole documented their work by only $12 \%$ partial necrosis in their 23 cases of intraoral reconstruction using the nasolabial flap. ${ }^{21,22}$

Regarding hair growth it was truly noticed in 12 patients in a small area at the base of the flap and disappeared spontaneously in 8 patients after receiving the radiotherapy. Nasolabial flap is thin but may interfere with wearing denture which was reported in 3 of our patients and debulking was done in secondary stage.

The reported major complications of the nasolabial flap procedure include total flap loss, flap dehiscence, bleeding, obstructive sialadenopathy and persistent fistula. ${ }^{23}$ In our series, we did not report any of these complications while using nasolabial flap.

\section{CONCLUSION}

Nasolabial Flap is a safe, simple, and reliable reconstruction option with adequate cosmetic and functional outcomes which can be used in small to moderate defect in orofacial region specially in older patients with comorbidities and contraindication to major surgery. 


\section{Conflict of Interest}

None

\section{Ethics statement/confirmation of patients' permission}

Done

\section{Funding}

This research received no external funding.

\section{REFERENCES}

1. A Ruiz-Moya, A Lagares-Borrego, P Infante-Cossío. Propeller facial artery perforator flap as first reconstructive option for nasolabial and perinasal complex defects. J Plast Reconstr Aesthet Surg 2015; 68(4):457-63

2. Prabhat K Bhama, Sapna A Patel, Umer Khan, Amit D Bhrany, Neal D Futran. Head and neck free flap reconstruction in patients older than 80 years. J Reconstr Microsurg 2014; 30(8):523-30

3. B Bianchi, A Ferri, S Ferrari, C Copelli, T Poli, E Sesenna. Free and locoregional flap associations in the reconstruction of extensive head and neck defects. Int J Oral Maxillofac Surg 2008; 37(8):723-9.

4. Y Ducic, M Burye. Nasolabial flap reconstruction of oral cavity defects: a report of 18 cases. J Oral Maxillofac Surg 2000;58(10):1104-8

5. Lekwale H, Zingade A (2016) Nasolabial flap reconstruction in Oral cavity cancer defects. Indian J Appl Res 6(2):535-7

6. Christopher M Harris, Robert Laughlin. Reconstruction of hard and soft tissue maxillofacial defects. Atlas Oral Maxillofac Surg Clin North Am. 2013; 21(1):127-38

7. James S Brown, Richard J Shaw. Reconstruction of the maxilla and midface: introducing a new classification. Lancet Oncol. 2010; 11(10):1001-8.

8. Urken ML, Moscoso JF, Lawson W, Biller HF. A systematic approach to functional reconstruction of the oral cavity following partial and total glossectomy Arch Otolaryngol Head Neck Surg. 1994 ;120(6):589-601

9. Eunkyu Lee, Song I Park, Donghyeok Kim, Hokyung Jin, Han-Sin Jeong . Modified bolster dressing with continuous suction improves skin graft survival for an oral cavity wound. J Otolaryngol Head Neck Surg. 2018 14;47(1):68

10. Tung-Yiu Wong, Ching-Hung Chung, Jehn-Shyun Huang, Hung-An Chen. The inverted temporalis muscle flap for intraoral reconstruction: its rationale and the results of its application. J Oral Maxillofac Surg . 2004; 62(6):667-75.
11. Stefano Spanio di Spilimbergo, Paolo Nordera, Samir Mardini, Giusy Castiglione, Harvey Chim, Vittore Pinna, Massimo Brunello, Claudio Cusino, Squaquara Roberto, Ugo Baciliero. Pedicled Temporalis Muscle Flap for Craniofacial Reconstruction: A 35-Year Clinical Experience with 366 Flaps. Plast Reconstr Surg. 2017; 139(2):468-76.

12. Mayank Tripathi, Sanjeev Parshad, Rajender Kumar Karwasra, Virender Singh. Pectoralis major myocutaneous flap in head and neck reconstruction: An experience in 100 consecutive cases. Natl J Maxillofac Surg. 2015; 6(1):37-41.

13. Thomas Mücke, Denys J Loeffelbein, Andreas Kolk, Stefan Wagenpfeil, Anastasios Kanatas, Klaus-Dietrich Wolff, David A Mitchell, Marco R Kesting. Comparison of outcome of microvascular bony head and neck reconstructions using the fibular free flap and the iliac crest flap. Br J Oral Maxillofac Surg. 2013; 51(6):514-9.

14. Lazaridis N, Zouloumis L, Venetis G, Karakasis D.The inferiorly and superiorly based nasolabial flap for the reconstruction of moderate-sized oronasal defects. J Oral Maxillofac Surg 56:1255-9.

15. E I Hofstra, S O P Hofer, J M Nauta, J L N Roodenburg, D H E Lichtendahl. Oral functional outcome after intraoral reconstruction with nasolabial flaps. Br J Plast Surg. 2004; 57(2):150-5.

16. Soh KBK, Soo KC. Reconstruction of moderate sized intraoral defects using the nasolabial flap. Ann Acad Med Singapore 1994; 23: 891-5.

17. Cornmck GC, Lamberty BGH. The Arterial Anatomy of Skin Flaps, 2nd ed. Edinburgh: Churchill Livingstone, 1994.

18. Ariyan S, Chicarilli ZN. Cancer of the upper aerodigestive system. In McCarthy JG, ed. Plastic Surgery, vol. 5. Philadelphia: WB Saunders Company, 1990: 3412-77.

19. Ioannides C, Fossion E (1991) Nasolabial flap for the reconstruction of defects of the floor of the mouth. Int $\mathrm{J}$ Oral Maxillofac Surg 20:40-3.

20. B T Varghese, P Sebastian, T Cherian, P M Mohan, I Ahmed, C M Koshy, S Thomas. Nasolabial flaps in oral reconstruction: an analysis of 224 cases. Br J Plast Surg. 2001; 54(6):499-503.

21. D C Herbert, J DeGeus. Nasolabial subcutaneous pedicle flaps. Br J Plast Surg. 1975; 28(2):90-6.

22. Mutimer KL, Poole MD. A review of nasolabial flaps for intra-oral defects. Br J Plast Surg 1987; 40:472- 7.

23. K L Mutimer, M D Poole. A review of nasolabial flaps for intra-oral defects. Br J Plast Surg. 1987; 40(5):472-7. 